\title{
IL RILANCIO DEL PROGETTO EUROPEO A 60 ANNI DAI TRATTATI DI ROMA
}

\author{
Nota del s.c. ALBERTO MARTINELLI (*)
}

(Adunanza del 6 aprile 2017)

\begin{abstract}
SuNTO. - Il saggio, scritto in occasione delle manifestazioni per il $60^{\circ}$ anniversario dei Trattati di Roma del 1957, ripercorre la storia del processo di integrazione, le sue conquiste e i suoi fallimenti, le sue crisi e le strategie del suo necessario rilancio. Esamina come l'accelerazione impressa al processo di integrazione in un'unione sempre più grande e diversificata sia stata possibile grazie a un compromesso tra il metodo di governance comunitario o sovranazionale delle politiche regolative del mercato unico e il metodo inter-governativo imperniato sul ruolo fondamentale del Consiglio europeo. Si critica il fatto che il processo di integrazione abbia trasferito porzioni crescenti di sovranità nazionale dal livello statale a quello sovranazionale senza un corrispondente trasferimento di impegno e lealtà alle istituzioni di una comunità sovranazionale in fieri da parte dei cittadini dei paesi membri che sono allo stesso tempo cittadini dell'Unione europea. Si analizzano le principali fratture oggi esistenti nell'UE, dovute alla diversità di obiettivi, interessi e prospettive dei paesi membri (tra chi considera l'UE un mero mercato unico e chi la concepisce anche come progetto politico sovranazionale; e tra i paesi creditori del Nord che considerano prioritario il rigore fiscale e i paesi debitori del Sud con economie in difficoltà, che si preoccupano soprattutto della stagnazione economica). E si mostra come queste fratture si siano approfondite a causa dell'intreccio di tre sfide: la sfida della crisi economico-finanziaria (con le sue implicazioni sociali di aumento delle disuguaglianze e della disoccupazione e sottoccupazione), la pressione migratoria e la minaccia del terrorismo fondamentalista. Si esamina, successivamente, la crescita dei partiti neo-nazionalisti e populisti euroscettici, riconducendola alla incompletezza e al deficit di legittimazione delle istituzioni rappresentative della democrazia europea e all'insufficiente sviluppo di una identità europea comune. Infine, si discutono le strategie di rilancio del progetto europeo, preferendo tra gli scenari delineati nel Libro Bianco del Presidente della Commissione quello orientato verso un'unione federale da perseguirsi mediante il metodo delle cooperazioni rafforzate.
\end{abstract}

(*) Professor Emeritus Università degli Studi di Milano, Italy.

E-mail: alberto.martinelli@unimi.it 
$* * *$

ABSTRACT. - Written on the occasion of the sixtieth anniversary of 1957 Treaties of Rome, the essay reviews the main stages of Europe's integration process, its achievements and failures, its crises and strategies for a necessary revival. The essay argues that the evolution toward an ever greater and diversified union was made possible by a compromise between the supranational-communitarian and the intergovernmental method of governance. The essay criticizes the fact that the transfer of quotas of national sovereignty to the supranational level has not be accompanied by an equivalent transfer of loyalty and commitment by member states' citizens, who are at the same time EU citizens. The main cleavages within the EU are then analysed, which can be traced to different goals, interest and expectations of member states: the cleavage between those who view the union just as a single market and those who conceive it also as a supranational politiical project; and the cleavage between Northern creditor countries who hold fiscal austerity as a policy priority and Southern indebted countries who are mostly concerned with economic stagnation and unemployment. And it is argued that these cleavages have deepened because of three interconnected challenges: the economicfinancial crisis and its social implications (growth of inequalities, unemployment and underemployment), the migration pressure, and the threat of fundamentalist terrorism. The essay then analyses the upsurge of national-populism and euroscepticism and traces these phenomena to the democratic deficit of EU institutions and the inadequate development of a common European identity. Finally, the alternative strategies for the EU renewal, ougtlined in the Commission's White Paper are discussed, arguing that the one oriented toward a federal union to be achieved through the method of reinforced cooperation is to be preferred.

\section{L'ANNIVERSARIO DEI TRATTATI DI ROMA}

Ormai spento il rumore delle manifestazioni per il $60^{\circ}$ anniversario dei Trattati di Roma, dominati da un eccesso di entusiasmi e di critiche, si può ora riflettere più pacatamente sullo stato attuale dell'Unione Europea, ripercorrendo rapidamente la storia del processo di integrazione, le sue conquiste e i suoi fallimenti, le sue crisi e le strategie del suo necessario rilancio, con riferimento a due documenti: la Dichiarazione dei capi di stato e di governo dell'Unione riuniti a Roma il 25 marzo e il Libro bianco sul futuro dell'Europa, presentato il $1^{\circ}$ marzo dal presidente della Commissione Jean Claude Juncker.

Va detto innanzitutto, prima di ogni doverosa analisi critica, che si tratta di una storia di successo. Di fronte alla chiassosa e rancorosa retorica anti-europeista che ha inondato i mass media in questi mesi (la UE come capro espiatorio di tutti i problemi presenti, le istituzioni europee come esempio di elite burocratiche e tecnocratiche, il tradi- 
mento dei valori fondanti del progetto europeo, 'l'Eurotta', l'eurofallimento', ecc.), è necessario riaffermare con convinzione che in 60 anni di vita, pur con tutti i suoi limiti e le sue questioni irrisolte, l'Unione Europea ha conseguito grandi obiettivi, ha rappresentato la principale innovazione politico-istituzionale della II metà del XX secolo, fondandosi su un nucleo di valori condivisi della cultura liberal-democratica e su interessi comuni garantiti dalle istituzioni dell'economia aperta (valori e istituzioni che definiscono i requisiti che devono dimostrare di possedere i paesi che intendono entrare). Il progetto europeo nasce dal desiderio di mettere fine a secoli di guerre civili europee, culminate nella immane tragedia e vergogna della Seconda Guerra mondiale, di garantire pace, libertà e sviluppo a popoli dissanguati e paesi semidistrutti in un contesto di nuove divisioni, rischi e conflitti (la guerra fredda, l'espansionismo sovietico, la minaccia nucleare). L'Unione ha promosso una crescita economica senza precedenti e sviluppato un sistema di welfare unico nel mondo (la UE ante-Brexit aveva meno del $7 \%$ della popolazione mondiale e il $22 \%$ del PIL mondiale, ma il 50\% della spesa per il welfare). Ha favorito il ritorno alla democrazia e consolidato i regimi liberal-democratici dei paesi europei che uscivano da esperienze autoritarie. Il nucleo originario dei sei paesi è cresciuto fino ad includerne 28 , caso unico nella storia di una entità politica che più che raddoppia la sua popolazione e il suo territorio senza colpo ferire.

Bene hanno fatto dunque i leader dei 27 paesi membri della UE riuniti a Roma il 25 marzo scorso a dichiararsi orgogliosi dei risultati raggiunti dall'Unione e della scelta coraggiosa e lungimirante che è alla sua origine. Ma perché i cittadini europei non festeggiano come dovrebbero (anche se per la prima volta i cortei a favore dell'integrazione europea sono stati più affollati di quelli degli euroscettici), perché molti di loro si dichiarano delusi e insoddisfatti, perché i britannici se ne vanno e qualcun altro vorrebbe uscire o addirittura disfare l'Unione?

Per rispondere bisogna esaminare, in primo luogo, come si è sviluppato il processo di integrazione, poi l'attuale intreccio di crisi e fratture e, infine, le alternative strategiche per il loro superamento.

\section{UN'UNIONE SEMPRE PIÙ GRANDE E DIFFERENZIATA}

L'integrazione europea è un processo in continua evoluzione, che ha coinvolto un numero crescente di paesi e ha costruito una architet- 
tura politico-istituzionale innovativa di grande complessità. Una storia di successo, come si è detto, ma segnata da contraddizioni, fratture e conflitti. Per comprendere le difficoltà presenti bisogna partire dalla constatazione che l'UE si è sempre più differenziata in virtù dell'acquisizione di un numero crescente di competenze e responsabilità a seguito di progressive adesioni, da 6 paesi fondatori (Francia, Germania, Italia, Olanda, Belgio e Lussemburgo) a 9 (con l'ingresso del Regno Unito, Irlanda e Danimarca nel 1973), a 10 (con l'adesione della Grecia nel 1981) a 12 (con l'ingresso di Spagna e Portogallo nel 1986) a 15 (con l'adesione di Svezia, Austria e Finlandia nel 1995, mentre in Norvegia l'adesione non venne ratificata dal referendum popolare) fino ai 28 stati membri dopo l'ingresso dei paesi dell'Est in questo secolo. Finchè l'integrazione ha riguardato essenzialmente l'abbattimento delle barriere doganali tra un gruppo limitato di paesi (i 6 fondatori più altri 6 nei successivi 30 anni) per realizzare un'area economica di libero scambio, le divergenze sono state contenute, la governance delle istituzioni sovranazionali è apparsa adeguata e i cittadini non hanno fatto mancare il loro consenso a un progetto di integrazione che appariva molto conveniente perché accresceva il loro benessere e la loro sicurezza. In questa fase si è andato affermando il metodo 'comunitario', in cui la Commissione ha il potere di iniziativa legislativa, il Consiglio europeo dei ministri e il Parlamento hanno un potere di co-decisione sulle proposte avanzate dalla Commissione e il Consiglio dei capi di stato e di governo è una sorta di presidenza collegiale dell'Unione' che ha la parola finale, mentre la Corte europea di giustizia dell'Unione Europea interpreta la normativa comunitaria e risolve le dispute tra i cittadini e gli stati membri. Tuttavia, man mano che il numero degli stati membri cresceva, il vestito confezionato per la comunità economica è risultato sempre meno adatto a un'unione più grande e con obiettivi più ambiziosi, un vestito sempre meno adatto non solo perché aumentava il numero dei paesi membri che aderivano con obiettivi solo parzialmente comuni, ma anche e soprattutto perché, a partire dalla seconda metà degli anni '80 del secolo scorso, si decise non solo di realizzare pienamente il mercato unico, ma di introdurre la moneta unica e di inserire nuove aree di policy nell'agenda politica europea, esemplificate dal Secondo e Terzo pilastro (esteri, difesa, sicurezza, giustizia e affari interni).

La forte accelerazione impressa al processo di integrazione è stata possibile grazie a un compromesso tra il metodo di governance comunitario o sovranazionale delle politiche regolative del mercato unico e il 
metodo inter-governativo imperniato sul ruolo fondamentale del Consiglio europeo, con il supporto della Commissione e la marginalizzazione del Parlamento. Questo compromesso, sancito dal Trattato di Maastricht del 1992 e codificato dal Trattato di Lisbona del 2007, ha in realtà aperto la strada a un crescente predominio del secondo sul primo. Le politiche del II e del III pilastro (difesa e ordine pubblico in particolare) sono, infatti, attributi intrinseci della sovranità statuale e riguardano questioni di interesse collettivo più direttamente rilevanti per il destino elettorale dei governi in carica, cosicché, anche per questo motivo, i governi nazionali non vogliono delegare in queste ambiti potere decisionale alle istituzioni sovranazionali. Si è verificato così il paradosso in base al quale la decisione di approfondire l'Unione voluta da coloro che erano più favorevoli all'integrazione politica (in prospettiva federale) ha comportato il predominio del metodo intergovernativo, rispetto a quello sovranazionale comunitario, rafforzando il potere dei governi nazionali e delle istituzioni comunitarie dove questo potere più direttamente si esplica, ovvero il Consiglio europeo.

Anche la creazione dell'Unione economica e monetaria (UEM) ha contribuito a questo rafforzamento, nonostante spostasse al livello sovranazionale un attributo fondamentale della statualità come la moneta. L'UEM è stata infatti il risultato di un ulteriore duplice compromesso: il primo è stato il compromesso tra gli stati che hanno aderito all'euro e gli stati che hanno scelto di non farlo (opting out) come i paesi scandinavi e il Regno Unito, che non ha comunque potuto evitare Brexit. Il secondo è il compromesso tra i due maggiori paesi dell'Eurozona, Germania e Francia: la prima voleva ottenere il consenso europeo alla riunificazione tedesca, la seconda voleva garantire l'ancoraggio europeo di una Germania più grande e potente e recuperare un certo grado di potere decisionale nella politica monetaria. Ne è derivata una centralizzazione sovranazionale della politica monetaria nella Banca centrale europea, e una stretta supervisione alle politiche macroeconomiche che restavano decentrate nei singoli stati membri, volta a verificare che rispettassero rigorosi vincoli di bilancio sanciti nel Patto di stabilità e crescita). Un compromesso che è fonte di costanti conflitti tra i governi degli stati membri e di faticosi negoziati tra questi e la Commissione europea.

La centralità del metodo intergovernativo e del Consiglio europeo nel processo decisionale dell'UE non deve stupire, perché il potere degli stati membri non è mai venuto meno. Non va dimenticato, infatti, 
che il processo di integrazione europea si è dispiegato sotto il controllo di stati nazionali consolidati con forti identità e ben definiti interessi. La maggior parte dei fondatori politici e intellettuali dell'Europa unita, pur ritenendo che le istituzioni europee dovessero estendersi progressivamente oltre i confini degli stati nazionali fino a sostituirsi ad essi, riconosceva realisticamente che il processo potesse avvenire solo sotto il loro controllo; una scelta realistica e probabilmente inevitabile, che ha garantito la sopravvivenza e resilienza degli stati membri in un mondo globalizzato, ma ha anche posto le basi per un risveglio dei nazionalismi, in tempi come quelli attuali di crisi economico-finanziaria, pressioni migratorie, attacchi terroristici e incertezza diffusa. Appare oggi evidente che egoismi nazionali, ideologie nazionaliste idiosincratiche, immagini stereotipate del carattere nazionale degli 'altri' europei sono dure a morire e contrappongono la loro forza emotiva agli argomenti razionali che propugnano una più profonda integrazione e sostengono che una unione sovranazionale costituisce il modo più efficace per gli stati di fronteggiare le nuove sfide della globalizzazione, poiché nessun paese membro, per quanto economicamente potente o politicamente ambizioso, può ragionevolmente pensare di agire da attore globale, mentre la UE possiede le risorse potenziali per farlo.

Questi argomenti razionali ('le ragioni dell'Unione') sono stati riaffermati nella dichiarazione di Roma del 25 marzo scorso dei leader dei 27 stati membri, ma molti cittadini appaiono disillusi, intimoriti e disorientati. Il nazionalismo blocca la strada verso il consolidamento dell'unione politica lo sviluppo di un'identità europea condivisa e l'adesione a un progetto comune che, a loro volta, potrebbero legittimare una governance autenticamente sovranazionale. Il calo di consensi per il progetto europeo è alimentato dalle crisi in corso (economica, dei migranti, del terrorismo), la cui gestione non è sufficientemente condivisa e distribuisce in modo diseguale costi e benefici tra i diversi paesi membri, esacerbando le disuguaglianze esistenti e i conflitti inter-statali e favorendo ideologie nazional-populiste che esigono il recupero della sovranità nazionale e la ri-nazionalizzazione di scelte politiche fondamentali. I conflitti di interesse tra stati sono più rilevanti dei conflitti tra classi. E i sentimenti di appartenenza sono tuttora molto più forti a livello nazionale che europeo.

L'eurodisaffezione e l'euroscetticismo sono riconducibili anche alla incompletezza e al deficit di legittimazione delle istituzioni rappresentative della democrazia europea. Nonostante gli sforzi per costruire 
un modello alternativo di democrazia multilivello e multi-stakeholder, infatti, il processo decisionale dell'Unione appare tuttora elitario e tecnocratico. I membri del Consiglio europeo, che costituisce il più importante organo decisionale nella UE, non sono scelti da un elettorato paneuropeo, ma traggono la loro legittimazione dai rispettivi elettorati nazionali e tendono quindi a porre l'interesse nazionale al di sopra dell'interesse comune. Come sostiene l'approccio funzionalista allo studio dell'integrazione europea, il trasferimento di quote di sovranità al livello sovranazionale è avvenuto spesso in modo 'spontaneo' e incrementale in base alla logica intrinseca dell'integrazione (tramite lo spillover funzionale e politico, l'integrazione negativa', il metodo di coordinamento aperto). In virtù della interdipendenza economica, dalla decisione di integrare alcuni settori deriva quella di integrarne altri e aumenta parallelamente l'esigenza di trasferire nuove competenze e decisioni politiche.

Il successo del mercato comune ha oscurato agli occhi di molti cittadini europei il fatto che il processo di integrazione è stato spesso promosso e sostenuto da elite economiche, politiche e intellettuali, con scarsa, ancorché crescente, partecipazione politica di massa. Finché vi è stata una chiara convenienza economica, il deficit di rappresentanza democratica è stato poco avvertito. Si è ritenuto che l'Unione fosse un esempio di output democracy, non di input democracy; ovvero che la sua legittimazione non derivasse tanto dalla piena applicazione del processo democratico (l'UE è infatti una democrazia rappresentativa di secondo grado perché il suo organo più importante, il Consiglio europeo, è formato dai ministri dei governi degli stati membri e la Commissione è nominata dai governi nazionali e solo in seguito approvata da Parlamento), quanto dai risultati della sua azione che sono ritenuti migliori di quelli conseguibili isolatamente dai singoli stati membri. Tuttavia, quando tra i cittadini di questo o quel paese membro si fa strada la percezione di crisi, sfide, risposte inadeguate o inique da parte della governance europea, cresce la protesta populista contro le 'elite burocratiche e tecnocratiche' di Bruxelles e Francoforte che 'tradiscono' la volontà dei popoli europei. Emerge allora la vulnerabilità di un processo che ha trasferito porzioni crescenti di sovranità nazionale dal livello statale a quello sovranazionale senza un corrispondente trasferimento di impegno e lealtà alle istituzioni di una comunità sovranazionale in fieri da parte dei cittadini di paesi membri diversi per obiettivi, interessi e aspettative. 
Il progressivo allargamento dell'Unione a gruppi di stati membri che in virtù di una diversa esperienza storica entrano nel processo di integrazione con diversi obiettivi e aspettative nei confronti del progetto comune e un diverso atteggiamento nei confronti della sovranità nazionale ha accresciuto le divergenze e i conflitti interni. Per stati come l'Italia e la Germania l'integrazione europea ha significato in primo luogo l'antidoto contro il ritorno al nazionalismo autoritario e totalitario. Il Regno Unito, i paesi scandinavi, e in buona misura anche la Francia, hanno condiviso il fondamentale obiettivo dell'unione come garanzia di pace, ma in contesto culturale in cui il nazionalismo democratico è stato una risorsa fondamentale per resistere e sconfiggere le aggressioni dei regimi non democratici. Ancora diverso è l'atteggiamento dei paesi post-comunisti dell'Est europeo, dove l'implosione dell'URSS ha rilanciato il nazionalismo come fonte di una rinnovata identità nazionale; questi paesi sono assai riluttanti a cedere porzioni della sovranità riacquistata dopo la fine del dominio sovietico, sia pure nel quadro di un progetto post-nazionale di un'unione democratica. Questa diversità di atteggiamenti verso la sovranità nazionale contribuisce a spiegare perché per il Regno Unito, i paesi scandinavi e dell'Europa dell'Est l'Unione significhi soprattutto mercato unico, mentre per gli altri significhi anche un progetto politico comune.

\section{LE FRATTURE E LE SFIDE}

Bisogna dunque prendere atto che l'UE è un'unione molto differenziata al suo interno, composta da stati con identità nazionali forti e una memoria storica comune e nel contempo diversa. Come rileva Fabbrini (2017), è opportuno riconoscere le diverse identità nazionali proprio per evitare la loro trasformazione in ideologie nazionalistiche anti-europee. Ma bisogna anche favorire la crescita di un senso di appartenenza all'Europa mediante adeguate politiche comuni (educative, sociali e culturali) e il rafforzamento della democrazia rappresentativa nell'Unione (Cavalli e Martinelli, 2015).

Ciò è tanto più necessario oggi in presenza di crisi che hanno accentuato le fratture e le divergenze derivanti dalla crescente differenziazione. Quattro sono le fratture principali: la prima frattura, tra Europa continentale e Regno Unito, si è chiusa con l'esito traumatico di Brexit. Ma il suo aspetto chiave, ovvero la contrapposizione tra una 
concezione dell'UE come mero mercato unico e quella dell'Unione come progetto politico sovranazionale permane nella seconda frattura, quella tra paesi occidentali di più antica appartenenza e paesi orientali di più recente ingresso nell'UE. La terza frattura contrappone i paesi creditori del Nord con economie più robuste-che considerano prioritario il rigore fiscale e rifiutano ogni forma di mutualizzazione del debito sovrano dei paesi più indebitati- ai paesi debitori del Sud con economie in difficoltà-che sono, per contro, preoccupati soprattutto della stagnazione economica e della elevata disoccupazione e rifiutano di tagliare la spesa pubblica (una frattura che si configura anche come contrapposizione tra la prospettiva dell'Europa neo-liberale e quella dell'Europa sociale). La quarta frattura, infine, riguarda la questione del libero movimento delle persone nello spazio europeo e coinvolge anche una componente crescente di immigrati e profughi, che è solo parzialmente identificabile con questo o quello stato nazionale; la popolazione immigrata accresce l'eterogeneità e le divisioni tra i governi dei paesi membri alimentando pregiudizi nazionalistici e chiusure xenofobe, richiederebbe una politica migratoria unica dell'Unione e produce invece politiche diverse da stato a stato, palesemente contradditorie nel caso di quei paesi che concepiscono l'UE come un mercato unico, ma vogliono conservare solo la libera circolazione di capitali e merci, non quella delle persone.

Le fratture oggi esistenti nell'UE, dovute alla diversità di obiettivi e prospettive dei paesi membri, si sono approfondite a causa dell'intreccio di tre sfide, la sfida della crisi economico-finanziaria (con le sue implicazioni sociali di aumento delle disuguaglianze, disoccupazione e sottoccupazione), la sfida del terrorismo fondamentalista e la sfida dei profughi e della pressione migratoria.

La prima sfida (la lunga crisi finanziaria-economica e del debito sovrano), è stata sostanzialmente vinta dall'UE con strategie efficaci, nonostante la lentezza e farraginosità dei suoi processi decisionali e il deficit di rappresentanza democratica delle sue istituzioni; ma le strategie adottate, pur efficaci nell'impedire il collasso del sistema monetario europeo e il fallimento degli stati più indebitati, ha comportato conseguenze negative, perché hanno accentuato le disuguaglianze sociali e territoriali tra i paesi membri (che fino al 2007 erano andate riducendosi anche in virtù della politica europea di coesione) e hanno alimentato divisioni tra stati del Nord e del Sud in merito alle politiche da attuare. Le politiche da attuare per riprendere la strada dello sviluppo e gover- 
nare il debito sovrano sono evidenti, ma assai impopolari, sia pure per ragioni diverse, e quindi si procede con lentezze, ritardi e in modo non coordinato, affidandosi al ruolo svolto dalla Banca centrale europea che ha tuttavia precisi limiti. Dalla crisi economico-finanziaria stiamo comunque uscendo, sia pur lentamente, ma con costi sociali e politici elevati che alimentano i partiti nazional populisti antieuropei.

La seconda sfida è quella del terrorismo fondamentalista e riguarda la sicurezza. Il terrorismo non è solo una minaccia esterna, ma ha radici anche nel cuore dell'Europa e mostra come i diversi modelli di integrazione degli immigrati, da quello assimilazionista francese a quello multiculturalista britannico, hanno parzialmente fallito, almeno per quella minoranza che rifiuta l'insieme di valori, istituzioni, atteggiamenti culturali, modi di vivere che definisce l'identità europea. E'una sfida che si può vincere, riaffermando le ragioni di una pacifica convivenza e di un comune destino che sono condivisi dalla maggioranza dei nuovi cittadini europei immigrati, e attuando efficaci politiche comuni di contrasto del terrorismo (politica di sicurezza e difesa comune) e di integrazione degli immigrati. Se ciò non avverrà, crescerà la protesta anti-europea del nazional-populismo.

La terza sfida, degli immigrati in cerca di lavoro e migliori condizioni economiche e dei profughi in fuga da guerre e violenze, è per certi aspetti la più difficile da affrontare e la più pericolosa, perché minaccia il fondamento stesso del progetto europeo, ovvero l'abbattimento delle frontiere nazionali e il superamento dei conflitti e degli egoismi nazionalistici. E'la più difficile da affrontare anche perché riacutizza le contraddizioni che hanno segnato il percorso della integrazione europea e il deficit di rappresentanza democratica dell'UE che ho esaminato in precedenza.

Si tratta di sfide distinte ma intersecate che, se non risolte, minacciano la sopravvivenza stessa dell'UE, in quanto suscitano incertezza, risentimento, paura, disaffezione tra i cittadini europei e mettono in discussione la capacità dei governi di soddisfare esigenze fondamentali di benessere socio-economico e di sicurezza individuale e collettiva, che sono anche condizioni necessarie per la conservazione della libertà e della coesione sociale. Sono sfide di tale entità che farebbe fatica ad affrontarle con successo uno stato sovrano ben consolidato, figurarsi una unione sovranazionale largamente incompleta.

Come rispondere a queste sfide, che rappresentano un terreno di coltura dei partiti e movimenti nazional-populisti anti-europei? 
(Martinelli, 2013, 2017). La risposta del nazional-populismo è quella di recuperare la piena sovranità nazionale, a cominciare dalla moneta e dal controllo delle frontiere, rinazionalizzare le politiche pubbliche oggi decise a livello sovranazionale, ovvero di fatto distruggere l'Unione, conservando al più qualche forma di libero scambio. E' una risposta non solo pericolosa perché risveglia nazionalismi latenti ma anche inefficace perchè si fonda sull'illusione di poter esercitare una piena sovranità statuale in un mondo globalizzato che erode la sovranità e condanna i diversi stati europei separati uno dall'altro all'irrilevanza nella politica mondiale o peggio alla loro sudditanza a una o all'altra delle grandi potenze.

\section{Come Rilanciare la UE E I CINQUE SCENARI DEL LIBRO BIANCO DELLA COMMISSIONE JUNCKER}

La sola risposta efficace alle crisi in corso è il rilancio dell'Unione, ovvero la costruzione di una autentica unione politica che sviluppi istituzioni democratiche rappresentative, affianchi alle politiche che sono già comuni (come quella della concorrenza e quella monetaria) altre politiche necessarie, come quella fiscale, migratoria, della sicurezza e della difesa e che sappia presidiare efficacemente la frontiera dell'Unione (è stato infatti un errore abolire i con fini interni tra gli stati membri senza presidiare i confini esterni).

La Dichiarazione dei capi di stato e di governo dell'Unione riuniti a Roma i 25 marzo 2017 va in questa direzione, anche se presenta la consueta discrasia tra obiettivi ambiziosi e riforme necessarie per conseguirli. I leader dei 27 stati membri si dichiarano infatti "orgogliosi per i risultati raggiunti dall'Unione europea", "un'unione unica dotata di istituzioni comuni e di forti valori... iniziata come il sogno di pochi e diventata la speranza di molti", che è il frutto di "un'impresa coraggiosa e lungimirante". Sono consapevoli delle sfide senza precedenti che l'Unione deve affrontare ("conflitti regionali, terrorismo, pressioni migratorie crescenti, protezionismo e disuguaglianze sociali ed economiche"). Promettono di rendere l'Unione europea "più forte e più resiliente, attraverso un'unità e una solidarietà ancora maggiori e nel rispetto di regole comuni. Si impegnano "a restare uniti" (perché è la migliore opportunità di influenzare le dinamiche mondiali e di difendere i nostri interessi e valori comuni) e "ad agire congiuntamente a ritmi e 
con intensità diversi se necessario, ma sempre nella stessa direzione, come abbiamo fatto in passato, in linea con i trattati e lasciando la porta aperta a coloro che desiderano associarsi successivamente". I leader sostengono il programma di Roma e si impegnano ad adoperarsi per realizzare a) "un'Europa sicura", determinata a combattere il terrorismo e la criminalità organizzata, in cui tutti i cittadini si sentano sicuri e possano spostarsi liberamente, con frontiere esterne protette e una politica migratoria efficace, responsabile e sostenibile; b) "un'Europa prospera e sostenibile che generi occupazione e sviluppo" con mercato e moneta unici, investimenti in innovazione, riforme strutturali volte al completamento dell'unione economica e monetaria; c) “un'Europa sociale sulla base di una crescita sostenibile", che favorisca il progresso economico e sociale, la coesione e la convergenza, l'integrità del mercato, tenendo conto delle diversità nazionali e del ruolo fondamentale delle parti sociali, e che promuova la parità tra donne e uomini e diritti e pari opportunità per tutti, la lotta contro la disoccupazione, la discriminazione, l'esclusione sociale e la povertà, migliori l'istruzione e la formazione, preservi il patrimonio culturale e promuova la diversità culturale; d) "un'Europa più forte sulla scena mondiale" che sviluppi i partenariati esistenti e nuovi, promuova stabilità e prosperità nelle altre regioni del mondo, si assuma maggiori responsabilità nella sicurezza e difesa comuni (inclusa l'industria della difesa) anche in cooperazione con la Nato, difenda il multilateralismo disciplinato da regole, e promuova un commercio libero ed equo e una politica climatica globale.

Il paragrafo conclusivo è il più deludente, perché la necessità di trovare un compromesso accettabile a tutti i 27 i paesi membri lo ha reso generico; si parla infatti di promozione di un processo decisionale democratico, efficace e trasparente, di dare ascolto e risposta alle preoccupazioni espresse dai cittadini, di dialogo con i parlamenti nazionali, di impegno a collaborare a tutti i livelli di governo con spirito di fiducia e leale collaborazione tra gli stati membri e tra essi e le istituzioni europee, di principio di sussidiarietà, di impegno a "promuovere un processo decisionale democratico, efficace e trasparente, e risultati migliori, di un'Unione grande sulle grandi questioni e piccola sulle piccole". Buoni propositi, obiettivi condivisibili, ma non si indica una strategia politica chiara per conseguirli. Se ci si limitasse al paragrafo conclusivo non si capirebbe quale strada i leader europei vogliono percorrere insieme, ma fortunatamente è rimasta nel documento la frase sui ritmi e le intensità diverse (se necessarie), che richiama la volontà espressa dai leader di 
Francia, Germania, Italia e Spagna pochi giorni prima dell'incontro di Roma di procedere nel percorso di un Europa a più velocità, corrispondente al terzo scenario del Libro bianco della Commissione denominato "Chi vuole di più fa di più".

Il Libro bianco (che si ricollega al Discorso di Juncker al Parlamento europeo sullo Stato dell'Unione del settembre 2016), dapprima, individua le aree critiche della UE (mercato unico e commercio, Unione monetaria, migrazioni e accordi di Shengen, politica estera e di sicurezza, bilancio comune $e$, in generale, efficacia attuativa dell'Unione stessa), per ognuna delle quali prevede l'elaborazione di un documento (reflection paper)- sotto la guida di uno o due presidenti insieme ai commissari interessati- in cui siano formulate proposte specifiche per il rilancio dell'Unione: il primo, previsto per il 26 aprile 2017, riguarderà la dimensione sociale dell'Europa e una proposta sul 'pilastro europeo dei diritti sociali' e verrà discusso a novembre nella conferenza di Goteborg; il secondo, previsto per metà maggio, avrà l'obiettivo di individuare metodi, regole e strumenti per controllare e valorizzare la globalizzazione, in vista del G7 a guida italiana di fine maggio e del G20 a guida tedesca di inizio luglio; il terzo documento, previsto per fine maggio riguarderà il rafforzamento dell'Eurozona alla luce del 'Rapporto dei cinque presidenti' pubblicato nel giugno del 2015; il quarto, previsto per inizio giugno, avrà per oggetto la difesa europea, in preparazione della Conferenza di Praga sullo stesso controverso tema; per fine giugno è previsto il documento sul bilancio dell'Unione. Infine, a settembre, i risultati del lavoro verranno riassunti nel discorso sullo Stato dell'Unione 2017 di Juncker.

Il Libro bianco delinea, poi, cinque scenari o percorsi (che potrebbero in certa misura anche combinarsi tra loro), l'impatto rispettivo sulle principali politiche (mercato unico e commercio, unione economia e monetaria, migrazione, sicurezza e accordo di Schengen, politica estera e di difesa, bilancio dell'UE) e sulla capacità di ottenere risultati, e la relativa road map per il futuro. Il primo scenario ("Avanti così), che prevede la continuazione del procedere a 'piccoli passi', giorno dopo giorno' col consenso di tutti, è chiaramente inadeguato a gestire efficacemente le crisi e il negoziato con il regno Unito dopo Brexit, perché ogni decisione rischia di essere paralizzata dalle divergenze tra gli stati membri o fortemente rallentata da faticosi compromessi al ribasso. Il secondo scenario ("Solo il mercato unico"), che prevede l'approfondimento della liberalizzazione del mercato e la rinazionalizzazione di alcune policies, 
configura di fatto un ritorno alla comunità economica europea prima di Maastricht con conseguente fine dell'euro, della BCE a della politica di difesa e sicurezza comune, e non è realisticamente accettabile dai paesi dell'Eurozona. Il terzo ("Chi vuole di più fa di più") è lo scenario dell'Europa a più velocità o a geometria variabile, e rappresenta un possibile compromesso tra i paesi che vogliono una maggiore integrazione politica e quelli che vogliono solo il mercato unico (come UK prima di Brexit e i paesi dell'Est europeo). Il quarto scenario ("Fare meno in modo più efficiente") ha un titolo suggestivo ma un contenuto poco chiaro; significa probabilmente concentrare il processo di integrazione su pochi obiettivi forti (commercio, moneta, difesa), rinunciando a quelli perseguiti con forme di integrazione più debole (come il metodo aperto di coordinamento) che riguardano in genere le politiche sociali. Il quinto ("Fare molto di più tutti insieme"), che fa riferimento a un'integrazione in tutti gli ambiti e configura quindi una completa unione federale, è l'opzione preferita dai federalisti, ma è oggi poco probabile perché richiederebbe una forte volontà di tutti gli stati membri. Dal momento che il primo scenario significa la mera continuazione dello status quo non è in grado di gestire adeguatamente le crisi attuali, il secondo e il quarto rischiano di approfondire gli squilibri e le fratture tra gli stati membri e il quinto è di assai difficile attuazione, lo scenario preferibile il terzo, l'unico che potrebbe realisticamente consentire di uscire dallo stallo presente e gestire le crisi in corso, senza esacerbare le divergenze esistenti tra gli stati membri. Questa scelta sembra condivisa dai leader dei quattro maggiori paesi dell'UE (dopo l'uscita del Regno Unito) e, sia pure con molta cautela, viene ribadita nel Documento di Roma sottoscritto da tutti i 27 stati membri.

Lo scenario dell'Europa a più velocità consente un rilancio dell'Unione; prende atto della differenziazione esistente e va nella direzione di un'unione federale (pur senza nominarla) con un nucleo duro di stati membri all'interno di una più ampia comunità economica plurale. E' lo scenario realisticamente preferibile, ma non è di facile attuazione. Il rilancio sarà reale se un gruppo di paesi (tutti quelli dell'Eurozona o gran parte di essi) decideranno di condividere in un'unione sovranazionale non solo le politiche regolative del mercato unico (condivise da tutti i 27 stati membri), non solo la moneta e la banca centrale (condivise dagli stati dell'Eurozona) ma anche altre fondamentali aree di policy. In primo luogo, la politica fiscale e di bilancio secondo le raccomandazioni fatte dal Gruppo di alto livello sulle risorse proprie, 
presieduto da Mario Monti (Commissione Europea, 'Il futuro finanziamento dell'UE', 2017) in cui, dopo aver affermato la necessità di intraprendere congiuntamente la riforma del bilancio e la riforma delle spese per rispondere alle nuove priorità dell'Europa contemporanea, si definiscono i principi guida della riforma (il valore aggiunto europeo, la sussidiarietà, l'onere fiscale complessivo inalterato per il contribuente europeo, le sinergie tra bilanci nazionali e bilancio europeo nella realizzazione degli obiettivi dell'Unione, la trasparenza e la leggibilità), e si propone, in particolare, di introdurre nuove risorse proprie (imposta sulle transazioni finanziarie, nuova IVA riformata, un'imposta sul $\mathrm{CO} 2$ ), consentendo di superare il sistema attuale dei contributi nazionali che acuiscono le tensioni tra gli stati membri 'contributori netti' e quelli che ricevono più di quanto versano. Anche se non si fa cenno al federalismo nel Rapporto, va rilevato che l'introduzione di una fiscalità autonoma dell'Unione è requisito essenziale per garantire la sua autonomia dagli stati membri.

In secondo luogo, politiche macroeconomiche e sociali comuni, che le risorse affluite direttamente al governo federale consentirebbero di attuare, finanziando interventi anticiclici, investimenti strategici in infrastrutture, ricerca e innovazione, nonché politiche per la salute, l'istruzione, l'edilizia popolare e altri interventi di politica sociale come un'assicurazione europea contro la disoccupazione, provvedimenti per l'integrazione sociale degli immigrati), e politiche ambientali e di risparmio energetico. Il governo dell'Unione dovrebbe anche dotarsi di un Tesoro europeo che possa emettere propri titoli per finanziarie le attività prioritarie e consentire una parziale mutualizzazione del debito degli stati membri.

Il terzo gruppo di politiche da attuare al livello sovranazionale è quello della politica estera e di sicurezza (controllo delle frontiere, esercito comune europeo, sistema integrato di intelligence, rete mondiale di rappresentanze diplomatiche).

In conclusione, tra i percorsi delineati dal Libro Bianco il terzo è l'unico che consenta realisticamente un rilancio dell'Unione. E la definizione dei gruppi di lavoro proposti sembrano individuare chiaramente le aree di policy in cui approfondire l'integrazione. La dichiarazione di Roma non delinea chiaramente questo percorso, ma vi accenna soltanto; è infatti un compromesso al ribasso, ma era difficile attendersi di più, prima degli appuntamenti elettorali che riguarderanno i tre più importanti paesi dell'UE a 27. I prossimi dodici mesi saranno decisivi, 
sia per il difficile negoziato con il Regno Unito che sarà un banco di prova per l'unità della UE, sia per le elezioni che si terranno nei maggiori stati membri. Il futuro dell'Unione non è messo a rischio dai risultati delle elezioni tedesche, in cui anche l'ingresso nel Bundestag del partito nazional-populista Alternative fur Deutschland non cambierebbe l'orientamento europeista del futuro governo, dato che europeisti sono sia i due partiti maggiori, la CDU di Angela Merkel e l'SPD di Martin Schulz, sia i loro eventuali alleati di governo. Cruciale sarà invece il risultato delle imminenti elezioni presidenziali francesi, in grado di fare la differenza tra sviluppo e crisi dell'Unione; infatti, la vittoria di Marine Le Pen, leader del Front National, potrebbe aprire la strada all'uscita della Francia dalla UE o comunque a un drastico indebolimento dell'Unione, mentre la vittoria di Emmanuel Macron, il più europeista tra i candidati alla presidenza, potrebbe ridare alla Francia il ruolo chiave che ha avuto in passato nella integrazione europea (come maggiore potenza politico-militare, che riequilibra la potenza economica della Germania), e rilanciare il progetto europeo a più velocità. Marine Le Pen appare sfavorita al ballottaggio contro Macron (come il moderatamente europeista Francois Fillion), ma dopo le fallaci previsioni su Brexit e Trump, nessun risultato si può dare per scontato.

L'influenza esercitata sul rilancio dell'UE dalle vicende politiche degli stati membri, in particolare i più grandi, non si limita tuttavia a Francia e Germania. Non vanno trascurate le elezioni italiane che si terranno probabilmente nella prossima primavera e che potrebbero produrre una situazione di difficile governabilità con conseguenze negative per l'intera Unione. Il sistema politico italiano è infatti tripolare con tre forze (PD, M5S, Lega, Forza Italia e FdI), non troppo diverse quanto a potenziale elettorale, che si dichiarano non disponibili ad allearsi al governo; e la legge elettorale maggioritaria modificata dalla Corte costituzionale configura un sistema proporzionale, il meno adatto a un contesto frammentato e difficilmente organizzabile intorno al polo che avrà la maggioranza relativa. Questo fatto, unitamente alla differenza con il sistema elettorale del Senato, rende assai probabile un risultato elettorale di ingovernabilità, simile a quella che si è verificata recentemente in Spagna. Se a questo si aggiungono le posizioni anti-euro e anti-UE di partiti che godono di ampi consensi, come il M5S e la Lega e il timido europeismo di altri, l'influenza della politica italiana potrebbe esser di ostacolo al rilancio dell'Unione

In conclusione, solo se le elezioni nei principali stati membri 
avranno esiti non sfavorevoli alle forze europeiste si potranno attuare le riforme necessarie per approfondire l'unione politica e conseguire gli obiettivi genericamente enunciati nella Dichiarazione di Roma in una prospettiva di Europa a più velocità, già attuata con la creazione dell'Unione monetaria, perché è chiaro da tempo ormai che l'idea di tenere tutti gli stati membri in un unico progetto di integrazione non funziona.

\section{BIBLIOGRAFIA}

Cavalli A. \& A. Martinelli, 2015, La società europea, Bologna, Il Mulino.

Commissione Europea, 2017, Libro bianco sul futuro dell'Europa, Bruxelles.

Commissione Europea, 2017, Il futuro finanziamento dell'UE:Rapporto finale $e$ Raccomandazioni del Gruppo di alto livello sulle risorse proprie.

Fabbrini S. 2017, Sdoppiamento.Una prospettiva nuova per l'Europa, Bari, Laterza.

Juncker J.C. 2016, Discorso al Parlamento europeo sullo Stato dell'Unione, Strasburgo.

Martinelli A. 2013, Mal di nazione. Contro la deriva populista, Milano, Università Bocconi Editore.

Martinelli A. 2016, Beyond Trump. Populism on the rise, Milano, Ispi.

Unione Europea, 2017,Dichiarazione dei capi di stato e di governo dell'Unione, Roma, 25.3.2017. 
